\title{
On Development of Meir-Keeler Type Fixed Point Theorems
}

\author{
K. Jha ${ }^{1}$, K.P.R. Rao ${ }^{2}$ and D. Panthi ${ }^{1}$ \\ ${ }^{1}$ Department of Natural Science (Mathematics), School of Science, \\ Kathmandu University, Dhulikhel, Nepal \\ ${ }^{2}$ Department of Applied Mathematics, Acharya Nagarjun University, \\ Nuzvid - 521 201, A.P., India \\ e -mail: jhakn(at)ku.edu.np
}

\begin{abstract}
The theory of fixed point is a very extensive field, which has various applications. The present paper deals with some developments of Meir-Keeler type fixed point theorem as its remarkable generalizations under several contractive definitions in metric space.
\end{abstract}

Key words: common fixed point, contraction, metric space, and compatible maps

\section{Introduction}

Fixed point theory is an important area of analysis. If $T$ is self mappings of a metric space $(X, d)$ then, a point $x$ in $X$ is said to be fixed point of $T$ if, $T x=x$; that is, a point which remains invariant under a self mapping is called a fixed point. In 1922, S. Banach proved a common fixed point theorem, which ensures under appropriate conditions, the existence and uniqueness of a fixed point. This result of Banach is known as the Banach contraction principle. This theorem provides a technique for solving a variety of applied problems in mathematical sciences and engineering. Many authors have extended, generalized and improved this result in different ways. The paper of Jha( 2002) is the survey work on some generalizations and applications of the Banach contraction principle. Also, the paper of Pant et. al.(2003) deals with the history of fixed point theorems.

We have the following definitions.

Definition 1. Let $(X, d)$ be a metric space and $T: X \rightarrow X$ be a mapping then $T$ is said to satisfy Lipschitz condition if, there exists a real number $k>0$ such that

$$
d(T x, T y) \leq k d(x, y) \text {, for all } x, y \text { in } X .
$$

Definition 2. A self-mapping $T$ of a metric space $(X, d)$ is called a weakly uniformly strict contraction or simply an $(\varepsilon-\delta)$ - contraction if for each $\varepsilon>0$ there exists $\delta>0$ such that for all $x, y \in X, \quad \varepsilon \leq d(x, y)<\varepsilon+\delta$ implies $d$ $(T x, T y)<\varepsilon$

Definition 3. Let $(X, d)$ be a metric space. The mappings $f$ and $g$ of $X$ are said to be $R$-weakly commuting if there exists a positive real number $R$ such that $d(f g x, g f x) \mathrm{d}$ " $R d(f x, g x)$, for all $x$ in $X$.

Definition 4. The mappings $f$ and $g$ are said to be $R$ weakly commuting of type $\left(A_{f}\right)$ if there exists a positive real number $R$ such that $d(f g x, g g x) \mathrm{d}$ " $R d(f x, g x)$, for all $x \in X$.

Definition 5. The mappings $f$ and $g$ are said to be $R$ weakly commuting of type $\left(A_{g}\right)$ if there exists a positive real number $R$ such that $d(g f x, f f x) \mathrm{d}$ " $R d(f x, g x)$, for all $x \in X$.

Definition 6. The mappings $A$ and $S$ are weakly commuting at point $x \in X$ whenever $d(A S x, S A x) \leq d(A x, S x)$. The pair $(A, S)$ is weakly commuting on $X$ if they commute at each point $x \in X$. It is noted that commuting maps are weakly commuting and the reverse implication is not true

Definition 7. Two self-mappings $A$ and $S$ of metric space $(X, d)$ are called compatible if, $\lim _{\mathrm{n}} d\left(A S x_{n}, S A x_{n}\right)=0$ 
whenever $\left\{x_{n}\right\}$ is a sequence in $X$ such that $\lim _{\mathrm{n}} A x_{n}$ $=\lim _{n} S x_{n}=t$ for some $t$ in $X$.

Definition 8. Two self mappings $A$ and $S$ of a metric space $(X, d)$ are called weakly compatible if they commute at coincidence points. That is, $A x=S x$ impies that $A S x=S A x$ for $x$ in $X$.

Definition 9. Two self maps $A$ and $S$ of a metric space ( $X$, d) will be called reciprocally continuous if $\lim _{n} A S x_{n}$ $=A t$ and $\lim _{n} S A x_{n}=S t$ whenever $\left\{x_{n}\right\}$ is a sequence such that $\lim _{n} A x_{n}=\lim _{n} S x_{n}=t$ for some $t$ in $X$.

Definition 10. Let $f$ and $g$ be self mappings of a set $X$. A point $u$ in $X$ is called a common fixed point of $f$ and $g$ if, $f u=u=g u$. Also, the point $\mathrm{u}$ is called a coincidence point of $f$ and $g$ provided $f u=g u$.

\section{Theorem 1. (Banach Contraction Principle)}

Any contraction mapping $T$ defined on a non-empty complete metric space $(X, d)$ into itself has a unique fixed point in $X$.

In 1969, A. Meir and E. Keeler proved a fixed point theorem for a weakly uniformly strict contraction mapping generalizing the Banach contraction principle as follows:

Theorem 2. If $(X, d)$ is a complete metric space. Then a weakly uniformly strict contraction $T$ on $X$ has a unique fixed point, say $u$, and for any $x \in X$, $\lim _{n \rightarrow \infty} T^{n} x=u$.

In 1978, Maiti and Pal established a fixed point theorem for self mapping satisfying the following generalization of weakly uniformly strict contraction (1): Given $\in>0$, there exists a $\delta>0$ such that

$\varepsilon \leq \max \{d(x, y), d(x, f x), d(y, f y)\}<\varepsilon+\delta$ implies $d$ $(f x, f y)<\varepsilon$.

Park and Rhoades in 1981, established fixed point theorems for pair of orbitally continuous self mappings of an orbitally complete metric space. Also, Park and Bae in 1981, extended the Meir-Keeler condition to commuting pair of self-maps in complete metric space. In 1984, Rhoades extended the result of Park and Rhoades (1981) involving a pair of mappings satisfying a Meir-Keeler type contractive definition into three mappings. Simultaneously, in 1984 Ganguli and in 1985
Rao and Rao extended Meir-Keeler type contractive definition into three mappings. In 1988, Fisher and Sessa proved the common fixed point for commuting pair of self mappings in complete metric with one of the mappings to be continuous. Pant in 1994 proved common fixed point for a pair of R-weakly commuting self mappings in complete metric space under contractive condition. Pathak et.al. in 1997 improved the results of Pant (1994) using R-weakly commuting self mappings of type $\left(A_{g}\right)$ or type $\left(A_{f}\right)$.

\section{Meir-Keeler Type fixed point theorems for two pairs of maps}

Jungck in 1986, obtained a common fixed-point theorem related to Meir-Keeler type contractive conditions for four continuous mappings on a compact metric space. As Meir-Keeler $(\varepsilon, \delta)$-contractive condition does not ensure a common fixed point unless $\delta$ satisfies some additional conditions or some additional inequalities, so, Pant in 1986 proved a common fixed point theorem for four continuous and commuting self mappings satisfying Meir-Keeler condition taking $\delta$ to be nondecreasing. Also, Rao and Rao in 1985 established a common fixed point theorem under Meir-Keeler type contractive conditions for four mappings. The most general common fixed theorems for four mappings, say $A, B, S$ and $T$ of a metric space $(X, d)$ use either Banach type contractive condition of the form

$$
\begin{aligned}
& d(A x, B y) \leq h m(x, y), \quad 0 \leq h<1 \text { where }(3) \\
& m(x, y)=\max \{d(S x, T y), d(\mathrm{Ax}, \mathrm{Sx}), d(B y, T y), \\
& [d(S x, B y)+d(A x, T y)] / 2\},
\end{aligned}
$$

or, a Meir-Keeler type $(\varepsilon, \delta)$ contractive condition of the form,

given $\varepsilon>0$, there exists $\delta>0$ such that

$\varepsilon \quad m(x, y)<\varepsilon+\delta \Rightarrow d(A x, B y)<\varepsilon$,

or, $a \phi$ contractive condition of the form

$d(A x, B y) \leq \phi(m(x, y)),(5)$

involving a contractive gauge function $\phi: R_{+} \rightarrow R_{+}$ is such that $\phi(t)<t$ for each $t>0$.

The weak form of the contractive condition (4) is of the form

$$
\varepsilon<m(x, y)<\varepsilon+\delta \Rightarrow d(A x, B y) \leq \varepsilon .
$$

Clearly, condition (3) is is a special case of both conditions (4) and (5).

The following example illustrates that an $(c, \delta)$ contractive condition of type (4) neither ensures the 
existence of a fixed point nor implies an analogous $\phi$ contractive condition (5).

Example 1. (Pant et.al 2002) Let $X=[0,2]$ and $d$ be the Euclidean metric on X. Define $f: X \rightarrow X$ by $f x=(1+x) / 2$ if, $x<1$ and $f x=0$ if $x \geq 1$. Then it satisfies the contractive condition

$$
\begin{aligned}
& \varepsilon \mathrm{d} " \max \{d(x, y), d(x, f x), d(y, f y),[d(x, f y)+d(f x, y)] \\
& / 2\}<\varepsilon+\delta \\
& \Rightarrow d(f x, f y)<\varepsilon, \text { with } \delta(\varepsilon)=1 \text { for } \varepsilon \geq 1 \text { and } \delta(\varepsilon) \\
& =1-\varepsilon \text { for } \varepsilon<1, \\
& \text { but } f \text { does not have a fixed point. Also, } f \text { does } \\
& \text { not satisfy the contractive condition } \\
& d(f \mathrm{x}, f \mathrm{y}) \leq(\max \{d(x, y), d(\mathrm{x}, f \mathrm{x}), d(y, f y), \\
& [d(x, f y)+\mathrm{d}(f x, y)] / 2\}),
\end{aligned}
$$$$
\text { since the desired function } \phi(t) \text { can not be defined at } t
$$
$=1$.

Hence, the two types of contractive conditions (4) and (5) are independent of each other. Thus, to ensure the existence of common fixed point under the contractive condition (4), the non decreasing (Pant 1994,1998), and lower semi continuous (Jungck 1986, Jungck et.al.1993) conditions on the function $\delta$ have been introduced. Jachymski (1994) has shown that the $(\varepsilon, \delta)$-contractive condition (4) with non decreasing $\delta$ implies a $\phi$-contractive condition (5). Also Pant et.al.(2002) have shown that $(c, \delta)$ - contractive condition (4) with a lower semi continuous $\delta$ implies a $\phi$-contractive condition (5). Thus, we see that if additional conditions are assumed on $\delta$ then the $(\varepsilon, \delta)$ - contractive condition (4) implies an analogous $\phi$-contractive condition (5) and both the contractive conditions hold simultaneously.

Theorem 3. (Pant 1986): Let $A, S$ and $B, T$ be commuting self mappings of a complete metric space $(X, d)$ satisfying $A X \subset T X, B X \subset S X$ and the condition: Given $\varepsilon>0$, there exists $\delta>0, \delta(\varepsilon)$ being non-decreasing such that $\varepsilon \leq \max \{\mathrm{d}(S x, T y), \mathrm{d}(A x, S x), \mathrm{d}(B y, T y)\}<\varepsilon+$ $\delta \mathrm{d}(A x, B y)<\varepsilon$.

If one of the mappings $A, B, S$ and $T$ is continuous, then $A, B, S$ and $T$ have a common fixed point.

In 1996, Singh and Chadha replaced the continuity condition from some of the main results of Jungck et.al.(1993) and established the following theorem.

Theorem 4. Let $(X, d)$ be a metric space, $S, T: X^{\prime} ! X$. Let $A, B$ be $(\varepsilon, \delta)-(S, T)$ contraction. If one of $A(X), B(X), S$ $(X)$ or $T(X)$ is a complete sub-space of $X$. Then,
$A$ and $S$ have a coincidence, and $\mathrm{B}$ and $T$ have a coincidence.

Further, if $A S u=S A u, u \in C(A, S)$ and $B T v=T B v$, $v \in C(B, T)$, then $A, B, S, T$ have a unique common fixed point, where $C(A, S)$ stands for a collection of coincidence points of $A$ and $S$; i.e. $C(A, S)=\{u: A u$ $=S u\}$.

Theorem 5. (Pant,2001): Let $(A, S)$ and $(B, T)$ be compatible pairs of self maps of a complete metric space $(X, d)$ such that $A X \subset T X, B X \subset S X$ and

(i) given $\varepsilon>0$ there exists a $\delta>0$ such that $\varepsilon$ d" $m(x, y)<\varepsilon+\delta \Rightarrow d(A x, B y)<\varepsilon$,

(ii) $d(A x, B y) d " \quad \phi(\max \{k d(S x, T y), d(A x, S x)$, $d(B y, T y), k[d(A x, T y)+d(B y, S x)] / 2\})$, where 1 d" $k<2$ and $\phi: R_{+}{ }^{\prime} ! R_{+}$such that $\phi(t)<\varepsilon$ for each $t>0$. If one of the mappings $A, B, S$ or $T$ be continuous then $A, B, S$ and $T$ have a unique common fixed point.

In 2003, Jha and Pant obtained following common fixed point theorem which employs a Lipschitz type analogue of known contractive definitions and provide a new type of answer to the open problem posed by B.E. Rhoades (1988) on the existence of a contractive definition.

Theorem 6. Let $(A, S)$ and $(B, T)$ be the compatible pairs of self mappings of a complete metric space $(X, d)$ such that

(i) $A X \subset T X, B X \subset S X$,

(ii) Given $\varepsilon>0$ there exists $\delta>0$ such that $\varepsilon \mathrm{d}$ " $m(x$, $y)<\varepsilon+\delta \Rightarrow d(A x, B y)<\varepsilon$, and

(iii) $d(A x, B y)<\max \{d(S x, T y), k[d(A x, S x)+d(B y$, $T y)] / 2$,

$[d(S x, B y)+d(A x, T y)] / 2\}, 1$ d" $k<2$.

If one of the maps $A, B, S$ and $T$ is continuous, then $A, B, S$ and $T$ have a unique fixed point.

In 2003, Jha et.al. established the following common fixed point theorem for two pairs of compatible mappings under a new contractive condition, which is independent of the known contractive definitions.

Theorem 7: Let $(A, S)$ and $(B, T)$ be compatible pairs of self mappings of a complete metric space $(X, d)$ such that 
(i) $A X \subset T X, B X \subset S X$,

(ii) Given $\varepsilon>0$ there exists a $\delta>0$ such that for all $x, y$ in $X, \varepsilon \mathrm{d}$ " $m(x, y)<\varepsilon+\delta \Rightarrow d(A x, B y)<\varepsilon$, and

(iii) $d(A x, B y)<k[d(S x, T y)+d(A x, S x)+d(B y, T y)$ $+d(S x, B y)+d(A x, T y)]$,

for $0 \mathrm{~d}$ " $k \mathrm{~d}$ " $1 / 3$. If one of the mappings $A, B, S$ and $T$ is continuous then $A, B, S$ and $T$ have unique common fixed point.

In 2004, Popa proved the following theorem for functions satisfying an implicit relation.

Theorem 8: Let $S, T, I$ and $J$ be the self mappings of a metric space $(X, d)$ such that (i) $S(X) \subset J(X)$ and $T(X)$ $\subset I(X)$,

(ii) given $\varepsilon>0$, there exists $\delta>0$ such that $\varepsilon \mathrm{d} " \max \{d(I x, J y), d(I x, S x), \mathrm{d}(\mathrm{Jy}, \mathrm{Ty}),[d(I x, J y)$ $+d(J y, T x)] / 2\}<\varepsilon+\delta$, implies $d(S x, T y)<\varepsilon$, and

(iii) there exists $F \in \mathbf{F}_{6}$, an implicit relation, such that inequality

$F(\mathrm{~d}(S x, T y), d(I x, J y), d(I x, S x), d(J y, T y) d(I x, T y)$, $d(J y, S x))<0$, where $F$ satisfies property $F(u)$ holds for all $x, y$ in $X$. If one of $S(X), T(X), I(X)$ and $J(X)$ is a complete subspace of $X$, then (a) $S$ and $I$ have a coincidence point, (b) $T$ and $J$ have a coincidence point. Moreover, if the pairs $(S, I)$ and $(T, J)$ are weakly compatible, then $S, T, I$ and $J$ have a unique common fixed point.

In 2004, Jha proved the following theorems for two pairs of compatible mappings.

Theorem 9: Let $(A, S)$ and $(B, T)$ be compatible pairs of self mappings of a complete metric space $(X, d)$ such that

(i) $A X \subset T X, B X \subset S X$,

(ii) Given $\varepsilon>0$ there exists a $\delta>0$ such that for all $x$, $y$ in $X$,

$\varepsilon<m(x, y)<\varepsilon+\delta \Rightarrow d(A x, B y) \mathrm{d}$ " $\varepsilon$, and (iii) $d(A x$,

$B y)<k[d(S x, T y)+d(A x, S x)+d(B y, T y)+d(S x, B y)$ $+d(A x, T y)]$,

for $0 d$ " $k \mathrm{~d}$ " $1 / 3$. If one of the mappings $A, B, S$ and $T$ is continuous, then $A, B, S$ and $T$ have a unique common fixed point.

Theorem 10.(Jha et.al 2005): Let (A, S) and $(B, T)$ be compatible pairs of self mappings of a complete metric space $(X, d)$ such that

(i) $A X \subset T X, B X \subset S X$, (ii) Given $\varepsilon>0$ there exists a $\delta>0$ s.t. for all $x, y$ in $X, \varepsilon$ $d " m(x, y)<\varepsilon+\delta \Rightarrow d(A x, B y)<\varepsilon$,

(iii) $d(A x, B y)<\max \left\{k_{1}[d(S x, T y)+d(A x, S x)+d(B y\right.$, $\left.T y)], k_{2}[d(S x, B y)+d(A x, T y)] / 2\right\}$, for $0 \mathrm{~d}^{\prime \prime} k_{1}<1,1$ d" $k_{2}<2$.

If one of the mappings $A, B, S$ and $T$ is continuous then $A, B, S$ and $T$ have unique common fixed point. In 2005, Jha et.al. proved the following common fixed point theorem employing a Lipschitz type analogue of known contractive definitions.

Theorem 11: Let $(A, S)$ and $(B, T)$ be compatible pairs of self mappings of a complete metric space $(X, d)$ such that

(i) $A X \subset T X, B X \subset S X$,

(ii) Given $\varepsilon>0$ there exists a $\delta>0$ such that, $\varepsilon \leq m(x$, $y)<\varepsilon+\delta \Rightarrow d(A x, B y)<\varepsilon$,

(iii) $d(A x, B y)<\max \left\{k_{l} d(S x, T y),[d(A x, S x)+d(B y\right.$, $\left.T y)] / 2, \mathrm{k}_{2}[d(\mathrm{Sx}, \mathrm{By})+\mathrm{d}(\mathrm{Ax}, \mathrm{Ty})] / 2\right\}$, for $\mathrm{k}_{1}>0$, $1 \mathrm{~d} " \mathrm{k}_{2}<2$.

If one of the mappings $\mathrm{A}, \mathrm{B}, \mathrm{S}$ and $\mathrm{T}$ is continuous, then $\mathrm{A}, \mathrm{B}, \mathrm{S}$ and $\mathrm{T}$ have a unique common fixed point. In 2007, Jha proved the following common fixed-point theorem involving two pairs of weakly compatible mappings under a Lipschitz type contractive condition.

Theorem 12: Let $A, B, S$ and $T$ be self mappings of a metric space $(X, d)$ such that

(i) $A X \subset T X, B X \subset S X$,

(ii) Given $\varepsilon>0$ there exists a $\delta>0$ such that for all $x, y$ in $X, \varepsilon<m(x, y)<\varepsilon+\delta \Rightarrow d(A x, B y) \mathrm{d}$ " $\varepsilon$, and

(iii) $d(A x, B y)<k[d(S x, T y)+d(A x, S x)+d(B y, T y)+d$ $(S x, B y)+d(A x, T y)]$, for $0 \mathrm{~d}$ " $k \mathrm{~d}$ " $1 / 3$. If one of the $A X, B X, S X$ and $T X$ is complete subspace of $X$ and if the pairs $(A, S)$ and $(B, T)$ are weakly compatible, then $A, B, S$ and $T$ have a unique common fixed point.

Theorem 13.(Rhoades et.al.1990) Let $(X, d)$ be a complete metric space, and $S, T$ self maps of $X$ with $S$ or $T$ continuous. Suppose there exists a sequence $\left\{A_{i}\right\}$ of self-maps of $X$ satisfying

(i) $A_{i}: X \rightarrow S X \cap T X$ for each $i$, or, (i') $S, T: X \rightarrow \cap_{i} A_{i} X$, (ii) each $A_{i}$ is compatible with $S$ and $T$, (iii) each $A_{i}$ weakly commutes with $S$ at each point $\xi$ for which $A_{i} \xi=S \xi$, and each $A_{i}$ weakly commutes with $T$ at each point $\eta$ for which $A_{i} \eta=T \eta$ and (iv) for any $\varepsilon>0$, there exists a $\delta$ 


\section{K. Jha et al./On Development of Meir-Keeler.}

such that, for each $x, y$ in $X, \varepsilon \leq M_{i j}(\mathrm{x}, \mathrm{y})<\varepsilon+\delta$ implies $d\left(A_{i} x, A_{j} y\right)<\varepsilon$, where $M_{i j}(x, y)=\max \left\{d(S x, T y) d\left(S x, A_{i} x\right)\right.$ $\left.d\left(T y, A_{j} y\right),\left[d\left(S x, A_{j} y\right)+d\left(T y, A_{i} x\right)\right] / 2\right\}$, then all $A_{i}, S$ and $T$ have a unique fixed point.

In 2001, Pant et.al. obtained the following fixed point theorem for a sequence of mappings satisfying Meir-Keeler type $(\varepsilon, \delta)$ contractive conditions. This theorem gives an answer to the question, which remained an open problem for more than a decade, on the existence of a contractive definition, which generates a fixed point but does not force the map to be continuous at the fixed point.

Let $\left\{A_{i}\right\}, \mathrm{i}=1,2,3 \ldots S$ and $T$ be self mappings of a metric space $(X, d)$. In the sequel, let us denote, $M_{1 i}(x$, $y)=\max \left\{d(S x, T y), d\left(A_{T} x, S x\right), d\left(A_{i} y, T y\right),\left[d\left(S x, A_{i} y\right)+\right.\right.$ $\left.\left.d\left(A_{T} x, T y\right)\right] / 2\right\}$.

We use this notation frequently in the following theorems for sequences of mappings.

Theorem 14: Let $\left\{A_{i}, i=1,2,3 \ldots\right\} S$ and $T$ be self mappings of a complete metric space $(X, d)$ such that

(i) $A_{1} X \subset T X, A_{i} X \subset S X, i>1$,

(ii) Given $\varepsilon>0$ there exists $\delta>0$ such that $\varepsilon \leq M_{12}(x, y)$

$<\varepsilon+\delta \Rightarrow d\left(A_{1} x, A_{2} y\right)<\varepsilon$

(iii) $d\left(A_{I} x, A_{i} y\right) \mathrm{d} " \varphi_{i}\left(M_{l i}(\mathrm{x}, \mathrm{y})\right), i>2$,

where $\phi_{i}: R_{+}{ }^{\prime} ! R_{+}$is such that $\phi_{i}(t)<t$ for each $t>0$. Let $A_{1}$ and $S$ be compatible and T be compatible with $A_{k}$ for each $k>1$. If one of the mappings is continuous then all the $A_{i}, S$ and $T$ have a unique common fixed point.

In 2002, Pant et.al. obtained a common fixed point theorem for a sequence of mappings under a weak form of $(\varepsilon, \delta)$-contractive condition and using a notion of reciprocal continuity of compatible pair of self-mappings on a complete metric space as follows.

Theorem 15: Let $\left\{A_{i}: i=1,2,3 \ldots\right\}, S$ and $T$ be self mappings of a complete metric space $(X, d)$ such that

(i) $A_{1} X \subseteq T X, A_{i} X \subseteq S X, i>1$,

(ii) Given $\varepsilon>0$ there exists $\delta>0$ such that $\varepsilon<M_{12}(x, y)$ $<\varepsilon+\delta \Rightarrow d\left(A_{T} x, A_{2} y\right) \leq \varepsilon$,

(iii) $d\left(A_{I} x, A_{i} y\right) d ” M_{l i}(x, y)$.

Let $S$ be compatible with $A_{1}$ and $T$ be compatible with $A_{k}$ for some $k>1$.If the mappings in one of the compatible pairs $\left(A_{l}, S\right)$ or $\left(A_{k}, T\right)$ are reciprocally continuous then all the $A_{i}, S$ and $T$ have a unique common fixed point.

In 2003, Pant et.al. obtained following fixed-point theorem for a sequence of mappings satisfying a weak form of Meir-Keeler type $(\varepsilon-\delta)$-contractive condition together with a $\phi$-contractive condition without assuming any additional conditions on $\delta$ and $\phi$. The theorem extends and unifies the Meir-Keeler type and a $\phi$-contractive type fixed point theorem.

Theorem 16: Let $\left\{A_{i}, i=1,2,3 \ldots\right\}, S$ and $T$ be self mappings of a complete metric space $(X, d)$ such that

(i) $A_{1} X \subset T X, A_{i} X \subset S X, i>1$

(ii) Given $\varepsilon>0$ there exists $\delta>0$ such that $\varepsilon<M_{12}$ $(x, y)<\varepsilon+\delta \Rightarrow d\left(A_{1} x, A_{2} y\right) \leq \varepsilon$

(iii) $d\left(A_{I} x, A_{i} y\right) \mathrm{d} " \phi_{i}\left(M_{1 i}(x, y)\right), i>2$,

where $\phi_{i}: R_{+}{ }^{\prime} ! R_{+}$is such that $\phi_{i}(t)<t$ for each $t>$ 0 .Let $A_{1}$ be compatible with $S$ and $T$ be compatible with $A_{k}$ for each $k>1$. If one of the mappings in a compatible pair is continuous then all the $A_{i}, S$ and $T$ have a unique common fixed point.

In 2007, Jha proved the following common fixed point theorem involving two pairs of weakly compatible mappings under a Lipschitz type contractive condition.

Theorem 17: Let $\left\{A_{\mathrm{i}}\right\}, \mathrm{i}=1,2,3 \ldots \mathrm{S}$ and $\mathrm{T}$ be self mappings of a metric space $(X, d)$ such that

(i) $A_{t} X \subset T X, A_{i} X \subset S X$ for $i>1$,

(ii) Given $\varepsilon>0$ there exists a $\delta>0$ such that for all $x, y$ in $X \varepsilon<M_{12}(x, y)<\varepsilon+\delta \Rightarrow d\left(A_{T} x, A_{2} y\right) \mathrm{d}$ " $\varepsilon$, and

(iii) $d\left(A_{T} x, A_{i} y\right)<\alpha\left[d(S x, T y)+d\left(A_{T} x, S x\right)+d\left(A_{i} y, T y\right)+\right.$ $\left.d\left(S x, A_{i} y\right)+d\left(A_{T} x, T y\right)\right]$, for $0 \mathrm{~d}$ " $\alpha \mathrm{d}$ " $1 / 3$.

If one of $A_{i} X, S X$ or $T X$ is complete subspace of $X$ and if the pairs $\left(A_{1}, S\right)$ and $\left(\mathrm{A}_{k}, T\right)$, for some $k>1$, are weakly compatible, then all the $A_{i}, S$ and $T$ have unique common fixed point.

\section{Remarks}

Theorem 14 gives a new generalization of Meir-Keeler type fixed point theorem for sequences and generalizes the results of Jungck (1986), Junck et. al.(1993), Pant $(1986,1993)$. The theorem does not require $\delta$ to be lower semi continuous or non-decreasing. Also, Theorem 15 improves the fixed point results due to Boyd and Wong (1969), Carbone et. al.(1989), Jachymski (1994), Jungck(1986), Jungck et. al.(1993), Matkoski (1975), Maiti and Pal (1978), Pant 
Nepal Journal of Science and Technology 10 (2009) 141-147

(1986,1993,1998), Park and Bae(1981) Park and Rhoades (1981) and Singh \& Kasahara (1982) Also, it can be noted that this theorem establishes a situation in which a collection of maps has a fixed point, which is a point of discontinuity. Moreover, Theorem 17 extends the result of Jha(2004,2007), Jha et.al. (2003,2005), Jha and Pant (2003), Pant and Jha $(2002,2003)$ and Pant et.al. $(2002,2003,2004)$ and improves the result of Popa (2005)

\section{Acknowledgement}

This paper is based on the M.Phil. thesis submitted to School of Science, Kathmandu University and it was presented at fifth National Conference on Science and Technology organized by NAST 2008. So, authors are thankful to both KU and NAST for their encouragement and to the referee for valuable remarks.

\section{References}

Boyd, D.W. and J.S. Wong.1969. On nonlinear contractions. Proc. Amer. Math. Soc., 20: 458-464.

Carbone, A., B.E Rhoades and S.P. Singh. 1989. A fixed point theorem for generalized contraction map. Indian J. Pure Appl. Math. 20: 543-548.

Cho, Y.J., P.P. Murthy and G. Jungck. 1993. A common fixed point theorem of Meir and Keleer type. Int. J. Math. Math. Sci. 16: 669-674.

Ciric, L.B. 1981. A new fixed point theorem for contractive mappings. Inst. Mathematique Publ., Nouvelle Serie. 30: $25-27$.

Fisher, B. and S. Sessa. 1988. Common fixed points for a pair of mappings. J. Univ. Kuwait (Sci.) 15: 9-11.

Ganguly, A. 1984. On an extension of a fixed-point theorem of Meir-Keleer for three mappings. Jnanabha 14: $157-161$.

Jachymshi, J. 1994. Common fixed point theorems for some families of mappings, Indian J. Pure Appl. Math. 25: 925- 937.

Jachymski, J. 1995. Equivalent conditions and Meir-Keeler type theorem. J. Math. Anal. Appl. 194: 293-303.

Jungck, G. 1986. Compatible mappings and common fixed points. Int. J. Math. Sci. 9: 771-779.

Jungck, G., K.B. Moon, S. Park and B.E. Rhoades. 1993. On generalizations of the Meir-Keeler type contractive maps: Correction. J. Math Anal. Appl. 180: 221-222.

Jha, K. 2002. Some applications of Banach contraction principle. Nepal. J. Sci. Tech. 4: 135-140.

Jha, K. and V. Pant. 2003. Ageneralization of unified common fixed-point theorem, The Nepali Math. Sci. Report 21: 62- 72 .

Jha, K., R.P. Pant and S.L. Singh. 2003. Common fixed points for compatible mappings in metric spaces. Radovi Math. 12: 107-114.
Jha, K. and R.P. Pant. 2003. A generalization of a Meir- Keeler type common fixed point theorem for compatible maps. Varahmihir J. Math. Sci. 3: 27-34.

Jha, K. 2004. A common fixed-point theorem for compatible mappings in metric space, Proceedings of IV National Conference on Sci. Tech.: 1565 - 1570.

Jha, K., R.P. Pant and S.L. Singh. 2005. On the existence of common fixed point for compatible mappings. Punjab Univ. J. Math. 37: 39-48.

Jha, K., R.P. Pant, and G. Porru. 2005. On Meir-Keeler type common fixed points for compatible maps. the Nepali Math. Sc. Report. 25: 29 - 34.

Jha, K. 2007. Common fixed point theorem for weakly compatible non-continuous mappings. Thai J. Math. 5: 191-197.

Jha, K. 2007. Common fixed point for weakly compatible maps in metric space. Kath. Univ. J. Sci. Eng. Tech. I: 1 - 6 .

Meir, A. and E. Keeler. 1969. A theorem on contraction mapping. J. Math. Anal. Appl. 28: 326-329.

Matkwoski, J. 1975. Integrable solutions of functional equations. Diss. Math. 127.

Maiti, M. and T.K. Pal. 1978. Generalization of two fixedpoint theorems. Bull. Calcutta Math. Soc. 70: 57-61.

Pant, R.P. 1986. Common fixed point of two pairs of commuting mappings. Indian J. Pure Appl. Math. 17: 187-192.

Pant, R.P. 1993. Common fixed point of weakly commuting mappings, Math. Student. 62: 97-102.

Pant, R.P. 1994. Common fixed points of non-commuting mappings. J. Math. Anal. Appl. 188: 436-440.

Pant, R.P. 1996. Common fixed point of sequence of mappings. Ganita. 47: 43-49.

Pant, R.P. 1998. Common fixed-point theorem for contractive mappings. J. Math. Anal. Appl. 226: 251-258.

Pant, R.P. and V. Pant. 1999. A Unified fixed-point theorem. Bull. Calcutta Math. Soc. 91: 227-232.

Pant, R.P. and V. Pant. 2000. Common fixed point under strict contractive condition. J. Math. Anal. Appl. 248: 327-332.

Pant, R.P., P.C. Joshi and V. Gupta. 2001. A Meir-Keleer type fixed point theorem. Indian J. Pure Appl. Math. 32: 779-787.

Pant, R.P. 2001. A generalization of contraction principle. $J$. Indian Math. Soc. 68: 25-32.

Pant, R.P. 2001. A new common fixed-point principle. Soochow J. Math. 27: 287-297.

Pant, R.P. and K. Jha. 2002. A generalization of Meir-Keeler type common fixed-point theorem for four mappings. J. Nat. Physical Sci. 16: 77-84.

Pant, R.P., A.B. Lohani and K. Jha. 2002. Meir-Keeler type fixed-point theorem and reciprocal continuity. Bull. Cal. Math. Soc. 94: 459-466.

Pant, R.P., A.B. Lohani and K. Jha. 2002. A history of fixed point theorems. Ganita Bharati. 24: 147- 159. 


\section{K. Jha et al./On Development of Meir-Keeler.}

Pant, R.P. 2003. Meir-Keeler type fixed point theorems and dynamics of functions. Demonostratio Math. 36: 199-206.

Pant, R.P. and K. Jha. 2003. A generalization of Meir Keeler type common fixed point theorem for four mappings. J. Ultra Sci. Phys. Sci. 15: 97-102.

Pant, R.P., K. Jha and A.B. Lohani. 2003. Generalization of a Meir-Keleer type fixed point theorem. J. Indian Math. Soc.70: 57-65.

Pant, R.P., K. Jha, V. Pant and V.P. Pande. 2004. A generalization of Meir-Keeler type common fixedpoint theorem. Ganita. 55: 87-93.

Park, S. and B.E. Rhoades. 1981. Extension of some fixed point theorems of Hegedus and Kasahara. Math. Seminar Notes. 9: 113-118.

Park, S. and B.E. Rhoades. 1981. Meir- Keeler type contractive conditions. Math. Japonica. 26: 13-20.

Park, S. and J.S. Bae.1981. Extension of a fixed point theorem of Meir Keeler. Ark. Math., 19: 223-228.

Park, S. and K.P. Moon. 1983. On generalized Meir-Keeler type conditions. Proc. Acad. Korea. 22: 31-41.

Pathak, H.K, Y.J. Cho and S.M. Kang. 1997. Remarks on R-weakly commuting mappings and common fixedpoint theorems. Bull. Korean Math. Soc. 34: 247- 257.
Popa, V. 2004. A general common fixed-point theorem of Meir and Keeler type for non-continuous weak compatible mapping. Filomat (Nis) 18: 33-40.

Popa, V. 2005. A generalization of Meir-Keeler type common fixed point theorem for four non-continuous mappings. Sarajevo J. Math. 1: 135-142.

Rao, I.H.N. and K.P.R. Rao. 1985. Generalization of fixedpoint theorems of Meir - Keleer type. Indian J. Pure Appl. Math. 16: 1249-1262.

Rhoades, B.E. 1984. A Meir-Keeler type fixed point theorem for three mappings. jnanabha 14:17-21.

Rhoades, B.E. 1988. Contractive definitions and continuity. Contemporary Math. 72: 233-245.

Rhoades, B.E., S. Park and K.B. Moon. 1990. On generalizations of the Meir-Keeler type contraction maps. J. Math. Anal. Appl. 146: 482 - 494.

Singh, S.L. and S. Kasahara. 1982. On some recent results on common fixed points. Indian J. Pure Appl. Math. 13: $757-761$.

Singh, S.L. and V. Chaddha. 1996. Remarks on recent fixed point theorems for compatible maps. Int. J. Math. Math. Sci. 19: 801-804.

Singh, S.L. and A. Tomar. 2003. Weaker forms of commuting maps and existence of fixed points. J. Korean Soc. Math. : Edu. Series B. Pure Appl. Math. 10: $145-161$. 
Nepal Journal of Science and Technology 10 (2009) 